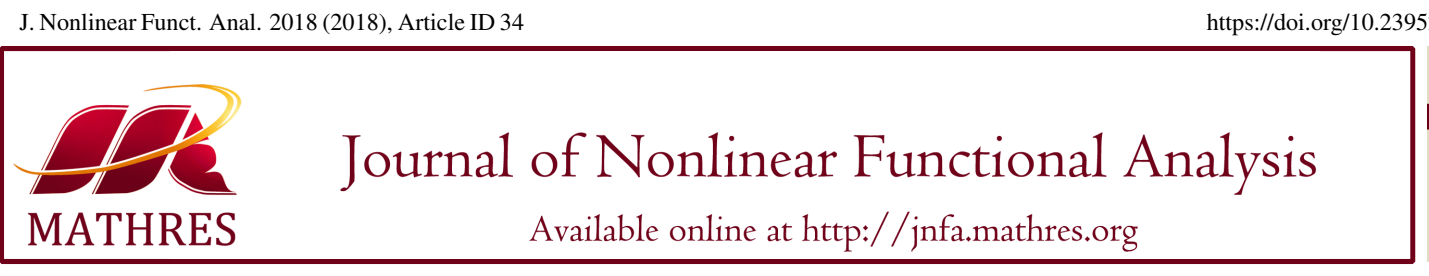

https://doi.org/10.23952/jnfa.2018.34

\title{
AUXILIARY PRINCIPLE TECHNIQUE FOR THE GENERAL LAX-MILGRAM LEMMA
}

\author{
THEMISTOCLES M. RASSIAS ${ }^{1}$, MUHAMMAD ASLAM NOOR $^{2, *}$, KHALIDA INAYAT NOOR $^{2}$ \\ ${ }^{1}$ Mathematics Department, National Technical University of Athens, Athens, Greece \\ ${ }^{2}$ Mathematics Department, COMSATS University Islamabad, Islamabad, Pakistan
}

\begin{abstract}
In this paper, we use the auxiliary principle technique to discuss the existence of a solution of the general LaxMilgram lemma. This technique is also used to suggest some new iterative methods. Convergence analysis of the proposed methods is considered under some mild conditions. Ideas and techniques of this paper may stimulate further research in this research filed.
\end{abstract}

Keywords. Absolute value equation; Lax-Milgram Lemma; Auxiliary principle; Iterative method; Convergence.

2010 Mathematics Subject Classification. 47J40, 49J40.

\section{INTRODUCTION}

The representation theorem states that a linear continuous functional can be represented by the inner product in a Hilbert space, the origin of which can be traced back to Riesz and Frechet. The inner product is a bilinear function. Then the question arises whether such a representation result holds for an arbitrary bifunction. The answer to this is affirmative. Lax-Milgram [1] proved that a linear continuous function can be representation by an arbitrary bifunction under suitable conditions. This representation is known as the Lax-Milgram Lemma and plays a significant role in the development of various branches of mathematical and engineering sciences. Motivated and inspired by ongoing research in this interesting field, we consider a general Lax-Milgram lemma, which can also be viewed as the weak formulation of the boundary value problems. It is shown that the Lax-Milgram Lemma [1] can be obtained as a special case. We use the auxiliary principle technique, which is mainly due to Lions and Stampachia [2] and Glowinski, Lions and Tremolieres [3], to discuss various aspects of the general Lax-Milgram Lemma. It is shown that the auxiliary principle technique can be used to suggest some iterative methods for solving the boundary value problems.

\footnotetext{
${ }^{*}$ Corresponding author.

E-mail addresses: trassias@ math.ntua.gr (T.M. Rassias), nooraslam@gmail.com (M.A. Noor), khalidan@gmail.com (K.I. Noor).

Received February 19, 2018; Accepted October 3, 2018.
}

(C) 2018 Journal of Nonlinear Functional Analysis 
In Section 2, we introduce the general Lax-Milgram Lemma and discuss its applications. The auxiliary principle technique is used to discuss the existence of the unique solution as well as to suggest some iterative methods for the boundary value problems. Convergence analysis of the proposed method is also considered under some mild conditions. As special cases, two new iterative methods for solving the boundary value problems are obtained.

\section{FORMULATIONS AND BASIC FACTS}

Let $H$ be a Hilbert space, whose norm and inner product are denoted by $\|\cdot\|$ and $\langle\cdot, \cdot\rangle$ respectively.

For a given operator $L$ and continuous functional $f$, we consider the problem of finding $u \in H$ such that

$$
\langle L u, v-u\rangle=\langle f, v-u\rangle, \quad \forall v \in H,
$$

which is called the general Lax-Milgram Lemma. This is also called the weak formulation of the boundary value problems. A wide class of problems arising in pure and applied sciences can be studied via equations (2.1).

If the bifunction $a(.,)=.\langle L u, v\rangle$, then the problem (2.1) is equivalent to finding $u \in H$ such that

$$
a(u, v)=\langle f, v\rangle, \quad \forall v \in H,
$$

which is known to be one of the most celebrated Lax-Milgram Lemma, see [1]. This result has been used to discuss the existence of a unique solution of the boundary value problems. This result is of tantamount significance in the study of function spaces and partial differential equations. For applications and generalizations of the Lax-Milgram Lemma, we refer the reader to [1, 2, 4, 5, 6, 7, 9, 10, 11, 12, 13] and the references therein.

If the operator $L$ is a linear, positive and symmetric, then problem (2.1) is equivalent to finding the minimum $u \in H$ of the functional $I[v]$ on $H$, where

$$
I[v]=\langle L v, v\rangle-2\langle f, v\rangle \quad \forall v \in H
$$

which is a quadratic programming problem and can be solved using the known techniques of the quadratic programming and optimization.

We now show that the boundary value problems can be studied via (2.1).

Example 2.1. Consider the boundary value problems of finding $u$ such that

$$
\frac{d^{2} u}{d x^{2}}=f(x), \quad \forall x \in[a, b]
$$

with boundary conditions

$$
u(a)=0, \quad u(b)=0
$$

where $f(x)$ is a continuous function, can be studied in the general framework of the problem (2.1). To do so, let $H_{0}^{1}[a, b]=\{u \in H: \quad u(a)=0, u(b)=0\}$ be a Hilbert space, see [3,9]. One can easily show 
that the energy functional associated with (2.1) is:

$$
\begin{aligned}
I[v] & =-\int_{a}^{b} \frac{d^{2} v}{d x^{2}} v d x-2 \int_{a}^{b} f v d x, \quad \forall u \in H_{0}^{1}[a, b] \\
& =\int_{a}^{b}\left(\frac{d v}{d x}\right)^{2}-2 \int_{a}^{b} f v d x \\
& =\langle L v, v\rangle-2\langle f, v\rangle .
\end{aligned}
$$

where

$$
\langle L u, v\rangle=\int_{a}^{b} \frac{d u}{d x} \frac{d v}{d x} d x
$$

It is clear that the operator $L$ defined by (2.6) is a linear, symmetric and positive. Therefore, the minimum of the functional $I[v]$ defined on the Hilbert space $H_{0}^{1}[a, b]$ can be characterized by equation (2.1). This shows that the boundary value problems can be studied in the framework of the problem (2.1).

We now recall some basic concepts and results.

Definition 2.2. An operator $L: H \longrightarrow H$ is said to be;

(i). Strongly monotone, if there exists a constant $\alpha>0$, such that

$$
\langle L u-L v, u-v\rangle \geq \alpha\|u-v\|^{2}, \quad \forall u, v \in H .
$$

(ii). Lipschitz continuous, if there exists a constant $\beta>0$, such that

$$
\|L u-L v\| \leq \beta\|u-v\|, \quad \forall u, v \in H
$$

(iii) monotone, if

$$
\langle L u-L v, u-v\rangle \geq 0, \quad \forall u, v \in H .
$$

We remark that, if the operator $L$ is both strongly monotone with constant $\alpha>0$ and Lipschitz continuous with constant $\beta>0$, respectively, then from (i) and (ii), it follows that $\alpha \leq \beta$.

\section{MAin RESUlts}

In this section, we use the auxiliary principle technique, the origin of which can be traced back to Lions and Stampacchia [1] and Glowinski,Lions and Tremolieres [3], as developed by Noor [5, 6, 8]. The main of idea of this technique is to consider an arbitrary auxiliary problem related to the original problem. In this way, one defines a mapping connecting the solutions of both problems. To prove the existence of a solution of the original problem, it is enough to show that this connecting mapping is a contraction which yields the solution of the original problem. Another novel feature of this approach is that this technique enables us to suggest some iterative methods for solving the boundary value problems.

Theorem 3.1. Let the operator $L$ be strongly monotone with constant $\alpha>0$ and Lipschitz continuous with constant $\beta>0$, respectively. If there exists a constant $\rho>0$ such that

$$
0<\rho \leq \frac{\alpha}{\beta^{2}},
$$

then there exists a unique solution of problem (2.1). 
Proof. (a). Uniqueness. Let $u_{1}=u_{2} \in H$ be two solutions of problem (2.1). Then

$$
\begin{aligned}
& \left\langle L u_{1}, v-u_{1}\right\rangle=\left\langle f, v-u_{1}\right\rangle, \quad \forall v \in H, \\
& \left\langle L u_{2}, v-u_{2}\right\rangle=\left\langle f, v-u_{2}\right\rangle, \quad \forall v \in H .
\end{aligned}
$$

Taking $\left.v=u_{2}\right)$ in (3.2) and $v=u_{1}$ in (3.3) and adding the resultant, we have

$$
\left\langle L u_{1}-L u_{2}, u_{1}-u_{2}\right\rangle=0 .
$$

Using the strongly monotonicity of $L$ with constant $\alpha>0$ and (3.4), we have

$$
\alpha\left\|u_{1}-u_{2}\right\|^{2} \leq\left\langle L u_{1}-L u_{2}, u_{1}-u_{2}\right\rangle=0
$$

from which, it follows that

$$
\alpha\left\|u_{1}-u_{2}\right\|^{2} \leq 0
$$

This implies that $u_{1}=u_{2}$, the uniqueness.

(b). Existence We now use the auxiliary principle technique to prove the existence of a solution of (2.1). To be more precise, for a given $u \in H$ satisfying (2.1), consider the problem of finding $w \in H$ such that

$$
\langle\rho L u, v-w\rangle+\langle w-u, v-w\rangle=\langle\rho f, v-w\rangle, \quad \forall v \in H,
$$

which is called the auxiliary problem, where $\rho>0$ is a constant. It is clear that (3.5) defines a mapping $w$ connected the both problems (2.1) and (3.5). To prove the existence of a solution of (2.1), it is enough to show that the mapping $w$ defined by (3.5) is a contraction mapping.

Let $w_{1} \neq w_{2} \in H \quad$ (corresponding to $\quad u_{1} \neq u_{2}$ ) be solutions of (3.5). Then

$$
\begin{array}{ll}
\left\langle\rho L u_{1}, v-w_{1}\right\rangle+\left\langle w_{1}-u_{1}, v-w_{1}\right\rangle=\left\langle\rho f, v-w_{1}\right\rangle, & \forall v \in H, \\
\left\langle\rho L u_{2}, v-w_{2}\right\rangle+\left\langle w_{2}-u_{2}, v-w_{2}\right\rangle=\left\langle\rho f, v-w_{2}\right\rangle, & \forall v \in H .
\end{array}
$$

Taking $v=w_{2}$ in (3.6) and $v=w_{1}$ in (3.7) and adding the resultants, we have

$$
\begin{aligned}
\left\|w_{1}-w_{2}\right\|^{2} & =\left\langle w_{1}-w_{2}, w_{1}-w_{2}\right\rangle \\
& =\left\langle u_{1}-u_{2}-\rho\left(L u_{1}-L u_{2}\right), w_{1}-w_{2}\right\rangle \\
& \leq\left\|u_{1}-u_{2}-\rho\left(L u_{1}-L u_{2}\right)\right\| \mid w_{1}-w_{2} \|,
\end{aligned}
$$

from which it follows that

$$
\left\|w_{1}-w_{2}\right\| \leq\left\|u_{1}-u_{2}-\rho\left(L u_{1}-L u_{2}\right)\right\| .
$$

Using the strongly monotonicity and Lipschitz continuity of the operator $L$ with constants $\alpha>0$ and $\beta>0$, we have

$$
\begin{aligned}
\left\|u_{1}-u_{2}-\rho\left(L u_{1}-L u_{2}\right)\right\|^{2}= & \left\langle u_{1}-u_{2}-\rho\left(L u_{1}-L u_{2}\right), u_{1}-u_{2}-\rho\left(L u_{1}-L u_{2}\right)\right\rangle \\
= & \left\langle u_{1}-u_{2}, u_{1}-u_{2}\right\rangle-2 \rho\left\langle L u_{1}-L u_{2}, u_{1}-u_{2}\right\rangle \\
& +\rho^{2}\left\langle L u_{1}-L u_{2}, L u_{1}-L u_{2}\right\rangle \\
\leq & \left(1-2 \rho \alpha+\rho^{2} \beta^{2}\right)\left\|u_{1}-u_{2}\right\|^{2} .
\end{aligned}
$$


Combining (3.9) and (3.11), we have

$$
\begin{aligned}
\left\|w_{1}-w_{2}\right\| & \leq\left(\sqrt{\left(1-2 \rho \alpha+\rho^{2} \beta^{2}\right)}\right)\left\|u_{1}-u_{2}\right\| \\
& =\theta(\rho)\left\|u_{1}-u_{2}\right\|
\end{aligned}
$$

where

$$
\theta(\rho)=\sqrt{1-2 \rho \alpha+\beta^{2} \rho^{2}} .
$$

We have to show that $\theta(\rho)<1$. It is clear that $\theta(\rho)$ assumes its minimum value for $\hat{\rho}=\frac{\alpha}{\beta^{2}}$ with $\theta(\rho)=\sqrt{1-\frac{\alpha^{2}}{\beta^{2}}}$. It follows that $\theta(\rho)<1$ for $0<\rho \leq \frac{\alpha}{\beta^{2}}$. Thus the mapping $w$ is a contraction mapping and consequently, it has a fixed point $w(u)=u \in H$ satisfying the problem (2.1).

Remark 3.2. We point out that the solution of the auxiliary problem (3.5) is equivalent to finding the minimum of the functional $I[w]$, where

$$
I[w]=\frac{1}{2}\langle w-u, w-u\rangle-\langle\rho(L u-f), w-u\rangle,
$$

which is a differentiable convex functional associated with inequality (3.5). This alternative formulation can be used to suggest iterative methods for solving the Lax-Milgram lemma. This auxiliary functional can also be used to find a kind of gap function, whose stationary points solves problem (2.1), see [4] and the references therein.

It is clear that, if $w=u$, then $w$ is a solution of (2.1). This implies that the auxiliary principle technique enables us to suggest the following iterative method for solving the problem (2.1).

Algorithm 3.3. For a given initial value $u_{0}$, compute the approximate solution $x_{n+1}$ by the iterative scheme

$$
\left\langle\rho L u_{n}+u_{n+1}-u_{n}, v-u_{n+1}\right\rangle=\left\langle\rho f, v-u_{n+1}\right\rangle, \forall v \in H .
$$

From Algorithm 3.3, one can easily obtain the Picard type iterative method for solving problem (2.1), which appears to be a new one.

Algorithm 3.4. For a given initial value $u_{0}$, compute the approximate solution $x_{n+1}$ by the iterative scheme

$$
u_{n+1}=u_{n}-\rho\left(L u_{n}-f\right), \quad n=0,1,2,3 \ldots
$$

We again use the auxiliary principle technique to suggest an implicit method for solving problem (2.1).

For a given $u \in H$ satisfying (2.1), consider the problem of finding $w \in H$ such that,

$$
\langle\rho(L w, v-w\rangle+\langle w-u, v-w\rangle=\rho\langle f, v-w\rangle, \quad \forall v \in H,
$$

which is called the auxiliary problem. We note that the auxiliary problems (3.5) and (3.12) are quite different. Clearly $w=u \in H$ is a solution of (2.1). This observation allows us to suggest the following iterative method for solving problem (2.1). 
Algorithm 3.5. For a given initial value $u_{0}$, compute the approximate solution $x_{n+1}$ by the iterative scheme

$$
\left\langle\rho L u_{n+1}+u_{n+1}-u_{n}, v-u_{n+1}\right\rangle=\left\langle\rho f, v-u_{n+1}\right\rangle, \forall v \in H,
$$

which is an implicit method.

From this implicit method, we can obtain the following iterative method for solving (2.1)

Algorithm 3.6. For a given initial value $u_{0}$, compute the approximate solution $x_{n+1}$ by the iterative scheme

$$
u_{n+1}=u_{n}-\rho\left(L u_{n+1}-f\right), \quad n=0,1,2,3 \ldots
$$

This is a new implicit method for solving problem (2.1).

To implement the implicit method, one uses the explicit method as a predictor and implicit method as a predictor. Consequently, we obtain the two-step method for solving problem (2.1).

Algorithm 3.7. For a given initial value $u_{0}$, compute the approximate solution $x_{n+1}$ by the iterative schemes

$$
\begin{aligned}
\left\langle\rho L u_{n}+y_{n}-u_{n}, v-u_{n+1}\right\rangle & =\left\langle\rho f, v-y_{n}\right\rangle, \forall v \in H, \\
\left\langle\rho L y_{n}+u_{n+1}-u_{n}, v-u_{n+1}\right\rangle & =\left\langle\rho f, v-u_{n+1}\right\rangle, \forall v \in H,
\end{aligned}
$$

which is known as two-step iterative method for solving problem (2.1).

Based on the above arguments, we can suggest a new two-step (predictor-corrector) method for solving problem (2.1).

Algorithm 3.8. For a given initial value $u_{0}$, compute the approximate solution $x_{n+1}$ by the iterative schemes

$$
\begin{aligned}
y_{n} & =u_{n}-\rho\left(L u_{n}-f\right) \\
u_{n+1} & =u_{n}-\rho\left(L y_{n}-f\right), \quad n=0,1,2 \ldots
\end{aligned}
$$

We now consider the convergence analysis of Algorithm 3.3 and this is the main motivation of our next result.

Theorem 3.9. Let $u \in H$ be a solution of (2.1) and let $u_{n+1}$ be the approximate solution obtained from Algorithm 3.3. If $L$ is a monotone operator, then

$$
\left\|u_{n+1}-u\right\|^{2} \leq\left\|u_{n}-u\right\|^{2}-\left\|u_{n+1}-u_{n}\right\|^{2}
$$

Proof. Let $u \in h$ be a solution of (2.1). Then

$$
\langle L u, v-u\rangle=\langle f, v-u\rangle, \quad \forall v \in H,
$$

implies that

$$
\langle L v, v-u\rangle \geq\langle f, v-u\rangle, \quad \forall v \in H,
$$

since $L$ is monotone. Taking $v=u_{n+1}$ in (3.15) and $v=u$ in (3.13), respectively, we have

$$
\left\langle L u_{n+1}, u_{n+1}-u\right\rangle \geq\left\langle f, u_{n+1}-u\right\rangle, \quad \forall v \in H,
$$


and

$$
\left\langle\rho L u_{n+1}+u_{n+1}-u_{n}, u-u_{n+1}\right\rangle=\left\langle\rho f, u-u_{n+1}\right\rangle, \forall v \in H .
$$

From (3.16) and (3.17), we have

$$
\left\langle u_{n+1}-u_{n}, u-u_{n+1}\right\rangle \geq 0 .
$$

It follows that

$$
\left\|u-u_{n+1}\right\|^{2} \leq\left\|u-u_{n}\right\|^{2}-\left\|u_{n}-u_{n+1}\right\|^{2},
$$

which is the required (3.14).

Theorem 3.10. Let $\bar{u} \in H$ be a solution of (2.1) and let $u_{n+1}$ be the approximate solution obtained from Algorithm 3.3. If $L$ is a monotone operator, then

$$
\lim _{n \rightarrow \infty} u_{n+1}=\bar{u} .
$$

Proof. Let $\bar{u} \in H$ be a solution of (2.1). From (3.14), it follows that the sequence $\left\{\left\|\bar{u}-u_{n}\right\|\right\}$ is noncreasing and consequently the sequence $\left\{u_{n}\right\}$ is bounded. Also, from (3.14), we have

$$
\sum_{n=0}^{\infty}\left\|u_{n+1}-u_{n}\right\|^{2} \leq\left\|u_{0}-\bar{u}\right\|^{2}
$$

which implies that

$$
\lim _{n \rightarrow \infty}\left\|u_{n+1}-u_{n}\right\|=0
$$

Let $\hat{u}$ be a cluster point of $\left\{u_{n}\right\}$ and the subsequences $\left\{u_{n_{j}}\right\}$ of the sequence $\left\{u_{n}\right\}$ converge to $\bar{u} \in H$. Replacing $u_{n}$ by $u_{n_{j}}$ in (3.13), taking the limit as $n_{j} \rightarrow \infty$ and using (3.20), we have

$$
\langle L \hat{u}, v-\bar{u}\rangle=\langle f, v-\hat{u}\rangle, \quad \forall v \in H,
$$

which shows that $\hat{u} \in H$ satisfies (2.1) and

$$
\left\|u_{n+1}-u_{n}\right\|^{2} \leq\left\|u_{n}-\hat{u}\right\|^{2} .
$$

From the above inequality, it follows that the sequence $\left\{u_{n}\right\}$ has exactly one cluster point $\hat{u}$ and $\lim _{n \rightarrow \infty} u_{n}=$ $\hat{u}$.

\section{CONCLUSION}

In this paper, we have considered a new approach for the Lax-Milgram lemma. The auxiliary principle technique has been used to study the existence of the unique solution of the boundary value problems. Some new iterative methods are considered. Convergence analysis of these iterative methods is investigated under suitable conditions. We would like to emphasize that the results obtained in this paper may motivate and bring a number of novel, potential applications, extensions and interesting new topics for further study.

\section{Acknowledgements}


The authors would like to express their sincere gratitude to the Rector, COMSATS University Islamabad, Pakistan, for providing excellent research facilities and academic environment.

\section{REFERENCES}

[1] P.D. Lax, A.N. Milgram, Parabolic Equations, contributions to the theory of partial differential equations, Ann. Math. Stud. 33 (1954), 167-190.

[2] J.L. Lions, G. Stampacchia, Variational inequalities, Commun. Pure Appl. Math. 20 (1967), 493-512.

[3] R. Glowinski, J.L. Lions, R. Tremolieres, Numerical Analysis of Variational Inequalities, North-Holland, Amsterdam. 1981.

[4] M. Fukushima, Equivalent differentiable optimization problems and descent methods for asymmetric variational inequality problems, Math. Program. 53 (1992), 99-110.

[5] M.A. Noor, On Variational Inequalities, PhD Thesis, Brunel University, London, 1975.

[6] K.I. Noor, M.A. Noor, A generalization of the Lax-Milgram lemma, Canad. Math. Bull. 23 (1980), 179-184.

[7] M.A. Noor, K.I. Noor, T.M. Rassias, Some aspects of variational inequalities, J. Comput. Appl. Math. 47 (1993), $285-312$.

[8] M.A. Noor, Variational inequalities and approximations, Punjab Univ. J. Math. 8 (1975), 25-40.

[9] L. Bers, F. John, M. Schechter, Partial Differential Equations, Academic Press, New York, 1966.

[10] H. Kozono, Lax-Milgram theorem in Banach spaces and its generalization to the elliptic system of boundary value problems, Manuscripta Math. 141 (2013), 637-662.

[11] W. Fechner, Functional inequalities motivated by the Lax-Milgram theorem, J. Math. Anal. Appl. 402 (2013), $411-414$.

[12] M.R. Galen, A version of Lax-Milgram theorem for locally convex spaces, J. Convex Anal. 16 (2009), 993-1002

[13] N. Nyamoradi, M.R. Hamidi, An extension of the Lax-Milgram theorem and its application to fractional differential equations, Electron. J. Differential Equations 2015 (2015), Article ID 95. 